\title{
A Simplified Approach to Students' Learning of Equivalent Solutions to Some Integral Calculus Problems
}

\author{
Mary Olukemi Odumosu, Philip Ajibola Bankole* \\ Department of Mathematics, Adeniran Ogunsanya College of Education, Lagos, Nigeria \\ Email address: \\ kemiodumosu@yahoo.com(M. O. Odumosu),pa.bankole@yahoo.com(P. A. Bankole) \\ ${ }^{*}$ Corresponding author
}

\section{To cite this article:}

Mary Olukemi Odumosu, Philip Ajibola Bankole. A Simplified Approach to Students' Learning of Equivalent Solutions to some Integral Calculus Problems. International Journal of Discrete Mathematics. Vol. 3, No. 1, 2018, pp. 21-27. doi: 10.11648/j.dmath.20180301.13

Received: March 31, 2018; Accepted: April 17, 2018; Published: May 10, 2018

\begin{abstract}
This paper presents simple approach to determine an Equivalent Solutions to some Integral Calculus Problems. An experimental study was carried out on one hundred and twenty (120) students offering Integral Calculus Course in the Department of Mathematics, Adeniran Ogunsanya College of Education, Otto/Ijanikin, Lagos State. The sample chosen includes male and female students from the following course combinations: Physics / Mathematics, Chemistry / Mathematics, Computer / Mathematics, Integrated Science / Mathematics, Economics / Mathematics, Biology/Mathematics and Geography / Mathematics. The students were grouped into seven based on their course combinations. The students in their respective groups were subjected to the same problem on integration where they are free to use any method of integration of their choice. The students came up with various solutions to a given integral calculus problem. Each of the solutions obtained in each group was evaluated on specified interval to determine the numerical value in order to draw inference on equivalent solutions. Maple software was used to determine if the solutions from each group are equivalent or differs. The numerical value and graphical representation of the solutions from each group using Maple software shows that the solutions obtained by the students in their respective groups are equivalent. Hence, maple software adaptation in teaching integral calculus enhanced the students' learning and by extension shows that equivalent solutions to some problems on integral calculus exists.
\end{abstract}

Keywords: Integral Calculus, Methods of Integration, Equivalent Solutions, Maple Software, Graphical Representation of Solutions

\section{Introduction}

Mathematics application is global in every facet of life. It is the heart of Science and Technology. The serviceable role of Mathematics in science and technology is complex and diverse in the sense that every area of science, technology and business enterprise is incomplete without mathematics. Mathematics including calculus is a primary doorway to engineering careers [1]. It was also described in [1] and cited in [2] as the mirror of civilization in all the centuries of painstaking calculation, and the most basic discipline for any person who would be truly educated in any science and in many other endeavours. The significance of Mathematics has made it indispensable in the curriculum in both Ordinary level and Advanced level of studies. There are numerous branches of Mathematics whereby Calculus is one of them with sub-divisions. Calculus as a branch of
Mathematics has very wide areas of applications in Physical Sciences and Engineering. An Integral Calculus became "unabridged" in the latter part of the seventeenth century as a result of the discovery of Isaac Barrow, Isaac Newton, and Gottfried Wilhelm Leibniz that integral of a function could be determined by asking what was differentiated to obtain that function [3]. Integral calculus depends on two fundamentally important concepts, that of a continuous function and that of the derivative of a continuous function. The Fundamental Theorem of Calculus connects the two branches of calculus: differential calculus and integral calculus. This study is restricted to integral calculus focusing on determining an equivalent set of solutions to a given integrable function over specified intervals.

Technology today has advanced to greater level through which a large number of today's students learn faster and easier compared to lecture method. [4]. Several Mathematical software that enhances the teaching and 
learning process in the classroom and outside the classroom are available such as Mathematica, Matlab, GeoGebra, Maple, etc. We apply Maple to enhance the teaching and learning process of integration after the group presentation of the solutions by the students on the given integration problems. Maple software was used to show graphical representation of the solution set obtained by the students in their respective groups. There was misconception among the students to ascertain the similarities/differences in the solutions obtained in their various groups.

\subsection{Focus of the Paper}

The focus of this paper is to present simple approach to determine if there is an equivalent solution set or difference in the integral of a given function. The method adopted to clarify that the solutions obtained by the students in their various groups are the same but with different look is the vital contribution of this research among other importance.

\subsection{Connection with Other Papers}

Many researches have been caried out on various aspects of integral calculus. This paper contributes to the existing literatures on integration of functions by presenting a simplified approach of conceptualizing the equivalent solution set to an integral problem. The two ways presented to determine equivalent solutions are: (i) numerical evaluation of the integral solutions over specified interval of integration after the application of various techniques of integration and (ii) Graphical representation of the solution set. All the graphical representation of the equivalent solutions set presented here were plotted using Maple.

The paper is organized as follow with section 1 Introduction, subsection 1.1 Focus of the Paper, subsection 1.2 Connection with other papers, Section 2 Preliminaries with subsections, Techniques of Integration of Functions, Fundamental Theorems of Calculus, Some Axioms of Definite Integral, Conditions for Integrality of functions, section 3 Methodology, section 4.Main Results, section 5 Result Discussion, and section 6 Conclusion.

\section{Preliminaries}

\subsection{Techniques of Integration of Functions}

Many researchers have contributed to the learning of methods of integration and applications in real life situations such as in [3-6]. Some of the methods of integration of function in existence are substitution method which centred on change of variables, integration by parts method for integrating product of functions, resolving into partial fraction method, resolving into Power series approach, numerical approximations of integrals such as: Simpsons rule, Trapezoidal rule, Riemann sum, Vegas method, Suave method, Divonne method, Cuhre method, etc. For the better understanding of various techniques of integration of functions, one can see $[5,6]$.

Definition 1: Any function $F$ such that $F^{\prime}(x)=f(x)$ is called a primitive, antiderivative, or indefinite integral of $f(x)$.

Theorem $1[3,8,9]$ : (Fundamental Theorem of Calculus). Let $f(x)$ be a differentiable function everywhere on $[a, b]$.

Then

$$
\int_{a}^{b} f(x) d x=F(b)-F(a) .
$$

Theorem 2 [3]: Any two antiderivatives $F$ and $G$ of $f(x)$ differ at most by a constant, that is,

$$
F(x)-G(x)=C .
$$

\subsection{Some Axioms of Definite Integral [7, 10]}

Let $f(x)$ and $g(x)$ be arbitrary integrable functions in an interval $[\mathrm{a}, \mathrm{b}]$, then the following axioms hold:

$$
\begin{gathered}
\int_{a}^{b}(f(x) \pm g(x)) d x=\int_{a}^{b} f(x) \pm \int_{a}^{b} g(x) d x \\
\int_{a}^{b} k f(x) d x=k \int_{a}^{b} f(x) d x
\end{gathered}
$$

where $k \neq 0$ is a constant.

$$
\int_{a}^{b} f(x) d x=\int_{a}^{c} f(x) d x+\int_{c}^{b} f(x) d x
$$

if $f(x)$ is integrable in $[a, c]$ and $[c, b]$.

$$
\begin{gathered}
\int_{a}^{a} f(x) d x=0 . \\
\int_{a}^{b} f(x) d x=\int_{a}^{b} f(t) d t \text { if } x=t . \\
\int_{a}^{b} f(x) d x=-\int_{b}^{a} f(x) d x \\
\int_{a}^{b} f(x) d x=\int_{a}^{b} f(a+b-x) d x . \\
\int_{0}^{b} f(x) d x=\int_{0}^{a} f(a-x) d x . \\
\int_{0}^{2 a} f(x) d x=\int_{0}^{a} f(x) d x+\int_{0}^{a} f(2 a-x) d x . \\
\int_{0}^{2 a} f(x) d x=2 \int_{0}^{a} f(x) d x
\end{gathered}
$$

provided that $f(2 a-x)=f(x)$ and $0, \quad$ if $f(2 a-x)=-f(x)$.

Suppose $f(x)$ is an even function, i.e $f(-x)-f(x)$, then

$$
\int_{-a}^{a} f(x) d x=2 \int_{0}^{a} f(x) d x
$$

For odd function, $f(-x)=-f(x)$, then 


$$
\int_{-a}^{a} f(x) d x=0
$$

Consider an interval $a \leq x \leq b, m \leq f(x) \leq M$ where $m$ and $M$ are constants, then we have

$$
m(b-a) \leq \int_{a}^{b} f(x) d x \leq M(b-a) .
$$

$$
\text { If } a \leq x \leq b, f(x) \leq g(x) \text {, then }\left|\int_{a}^{b} f(x) d x\right| \leq \int_{a}^{b}|f(x)| d x
$$
if

$$
a \leq b .
$$

\subsection{Conditions for Integrability of Functions}

There are various aspects of integral calculus. Conditions attached to some integrals depend on the kind of integral calculus one is dealing with.

Integral calculus depends on two fundamental important concepts: (i) continuous function and (ii) derivative of a continuous function. In addition to the above stated conditions, it is necessary that the function is well defined. Therefore, if a function is continuous on a given interval, then it's integrable on that interval. Also, if a function has only a finite number of some kinds of discontinuities on an interval, it is also integrable on that interval. Hence, every differentiable function is integrable and by extension continuous but the converse is not always true since some functions may be integrable but not differentiable at some points. For example, a function $y=|f(x)|$ is nondifferentiable at $x=0$ but integrable for all values of $\mathrm{x}$. The reader can see $[7,10]$.

Theorem 3 [3]: Let $f(x)$ be a function bounded in interval $[a, b]$, then a necessary and sufficient condition for the existence of $\int_{a}^{b} f(x) d x$ is that the set of discontinuities of $f(x)$ have measure zero.

\section{Methodology}

An experimental study was carried out on year two (200 Level) students in Adeniran Ogunsanya College of Education, Otto / Ijanikin, Lagos State, Nigeria. The students offer MAT 211 titled Integral Calculus in Mathematics Department involving male and female from the following course combinations: Physics / Mathematics, Chemistry / Mathematics, Computer / Mathematics, Integrated Science / Mathematics, Economics / Mathematics, Biology / Mathematics and Geography / Mathematics. The students were grouped into seven based on their course combinations. Some Problems on integration which has to do with indefinite integral were given to the students to solve as a group work. The students in their respective groups were subjected to the same problems on integration where they are free to use any method of integration of their choice. The students came up with various solutions to a given integral calculus problem in their respective groups.

The question now is how to determine if the solutions are correct in the actual sense or not, equivalent or differs from each other.

\section{Main Results}

For illustration, we consider a simple problem on integration among many other problems in what follows.

Problem 1.

Use any integration technique of your choice to determine the solution of $\int \sin (x) \cos (x) d x$.

\subsection{Group A Solution}

Using integration by Parts Method.

$$
\int u d v=u v-\int v d u
$$

Let $v=\sin x$, and $d v=\cos x$. Then $d u=\cos x d x$ and $v=\int d v=\int \cos x d x=\sin x$.

Substituting into the equation (1), we have:

$$
\begin{gathered}
\int \sin x \cos x d x=\sin x \cdot \sin x-\int \sin x \cdot \cos x d x \\
=\sin ^{2} x-\int \sin x \cdot \cos x d x
\end{gathered}
$$

This implies that

$$
2 \int \sin x \cos x d x=\sin ^{2} x
$$

Hence,

$$
\int \sin x \cos x d x=\frac{1}{2} \sin ^{2} x+c
$$

where $c$ is a constant of integration.

\subsection{Group B Solution}

By using substitution Method to carry out $\int \sin x \cos x d x$. Let $u=\cos x$. Then

$$
d u=-\sin x d x \Rightarrow d x=\frac{d u}{-\sin x} d x
$$

Then,

$$
\begin{gathered}
\int \sin x \cos x d x=\int \sin x \cdot u \cdot \frac{d u}{-\sin x} \\
\int \sin x \cos x d x=-\int u d u \\
\int \sin x \cos x d x=-\frac{u^{2}}{2}+c
\end{gathered}
$$




$$
\begin{aligned}
& \int \sin x \cos x d x=-(\cos x)^{2} / 2+c \\
& \int \sin x \cos x d x=-\frac{\cos ^{2} x}{2}+c \\
& \int \sin x \cos x d x=-\frac{\cos ^{2} x}{2}+c
\end{aligned}
$$

\subsection{Group C Solution}

Using substitution method with different choice of variable change. Let the solution of the integration problem be $y_{2}^{*}$. Now, set $u=\sin x \rightarrow d u=\cos x d x$. This implies that $d x=\frac{d u}{\cos x}$. Therefore,

$$
\begin{gathered}
\int \sin x \cos x d x=\int u \cdot \cos x \cdot \frac{d u}{\cos x} \\
\int \sin x \cos x d x=u d u \\
\int \sin x \cos x d x=\frac{u^{2}}{2} \\
\int \sin x \cos x d x=\frac{\sin ^{2} x}{2}+c
\end{gathered}
$$

Hence, the solution $y_{2} *$ coincide with $y_{1}$ in Group A but what can be said of the solution $y_{2}$ ? Before we delve into the analysis of the solution, let us consider the solution obtained by another group in what follows.

\subsection{Group D Solution}

Applying Trigonometrical identity of sine of double angle formula.

$$
\sin (2 x)=\sin (x+x)=2 \sin x \cos x .
$$

Therefore,

$$
\sin x \cos x=\frac{1}{2} \sin (2 x)
$$

Integrating the both sides wrt $\mathrm{x}$, we have

$$
\begin{gathered}
\int \sin x \cos x d x \equiv \int \frac{1}{2} \sin (2 x) d x=\frac{1}{2} \int \sin (2 x) d x \\
\int \sin x \cos x d x \equiv \frac{1}{2}\left(\frac{-\cos 2 x}{2}\right)+c . \\
\int \sin x \cos x d x=-\frac{1}{4} \cos 2 x+c .
\end{gathered}
$$

Hence, the solution $y_{3}=-\frac{1}{4} \cos 2 x+c$. The question asked by the students was that which of the solution set $\mathrm{y}_{1}$, $y_{2}$ and $y_{3}$ is correct? In order to provide answer to the students' question, The following two steps were applied.

Step 1: We evaluated each of the solution set on the same interval of integration. That is, we changed the problem to definite integral form and evaluate each of the solution to obtain numerical value.

Step 2: We graphed the solution set to the integration problem 1 on the same interval to determine the behaviour of the solution. The results are presented in sequel.

Assuming the solution exists on the interval $x \in\left[\frac{\pi}{4}, \frac{\pi}{2}\right]$. Then, for solution $y_{1}=\int \sin x \cos x d x=\frac{1}{2} \sin ^{2} x+c$, we have:

$$
\begin{gathered}
\int_{\frac{\pi}{4}}^{\frac{\pi}{2}} \sin x \cos x d x=\left.\left[\frac{1}{2} \sin ^{2} x+c\right]\right|_{\frac{\pi}{4}} ^{\frac{\pi}{2}} \\
\int_{\frac{\pi}{4}}^{\frac{\pi}{2}} \sin x \cos x d x=\frac{1}{2}\left[\sin ^{2}\left(\frac{\pi}{2}\right)+c-\left(\left(\sin ^{2}\left(\frac{\pi}{4}\right)+c\right)\right)\right] \\
\int_{\frac{\pi}{4}}^{\frac{\pi}{2}} \sin x \cos x d x=\frac{1}{2}\left[\sin ^{2}\left(90^{0}\right)-\sin ^{2}\left(45^{0}\right)\right] \\
\int_{\frac{\pi}{4}}^{\frac{\pi}{2}} \sin x \cos x d x=\frac{1}{2}\left[1-\left(\frac{1}{\sqrt{2}}\right)^{2}\right] \\
\int_{\frac{\pi}{4}}^{\frac{\pi}{2}} \sin x \cos x d x=\frac{1}{2}\left(1-\frac{1}{2}\right) \\
\int_{\frac{\pi}{4}}^{\frac{\pi}{2}} \sin x \cos x d x=\frac{1}{2} \times \frac{1}{2} \\
\text { Hence, } \int_{\frac{\pi}{4}}^{\frac{\pi}{2}} \sin x \cos x d x=\frac{1}{4}
\end{gathered}
$$

Similarly, consider the solution

$$
y_{2}=\int \sin x \cos x d x=-\frac{\cos ^{2} x}{2}+c .
$$

Evaluating the solution on the same interval of integration $x \in\left[\frac{\pi}{4}, \frac{\pi}{2}\right]$, we have:

$$
\begin{gathered}
y_{2}=\int_{\frac{\pi}{4}}^{\frac{\pi}{2}} \sin x \cos x d x=\left[-\frac{1}{2} \cos ^{2} x+c\right]_{\frac{\pi}{4}}^{\frac{\pi}{2}} \\
y_{2}=-\frac{1}{2}\left[\cos ^{2}\left(\frac{\pi}{2}\right)+c-\left(\cos ^{2}\left(\frac{\pi}{4}\right)+c\right)\right] \\
y_{2}=-\frac{1}{2}\left[\cos ^{2}\left(90^{0}\right)-\cos ^{2}\left(45^{\circ}\right)\right]
\end{gathered}
$$




$$
\begin{gathered}
y_{2}=-\frac{1}{2}\left[0-\left(\frac{1}{\sqrt{2}}\right)^{2}\right] \\
y_{2}=-\frac{1}{2} \times-\frac{1}{2}
\end{gathered}
$$

Hence $y_{2}=\frac{1}{4}$, which coincides with the solution $y_{1}$.

Finally, we evaluated the solution $y_{3}=-\frac{1}{4} \cos 2 x+c$ on the same integration interval $x \in\left[\frac{\pi}{4}, \frac{\pi}{2}\right]$ as follows.

$$
\begin{gathered}
y_{3}=\int_{\frac{\pi}{4}}^{\frac{\pi}{2}} \sin x \cos x d x \\
y_{3}=-\frac{1}{4}\left[\cos 2\left(\frac{\pi}{2}\right)+c-\left(\cos 2\left(\frac{\pi}{4}\right)+c\right)\right] \\
y_{3}=-\frac{1}{4} \cos \pi-\cos \left(\frac{\pi}{2}\right) \\
y_{3}=-\frac{1}{4}\left(\cos 180^{0}-\cos 90^{\circ}\right) \\
y_{3}=-\frac{1}{4}(-1-0)
\end{gathered}
$$

Graphical Representation of the Equival ent Solutions y_1, y_2 and $\mathrm{y}_{-} 3$ of the Integration Problem 1 on the interval $[\pi / 4, \pi / 2]$.

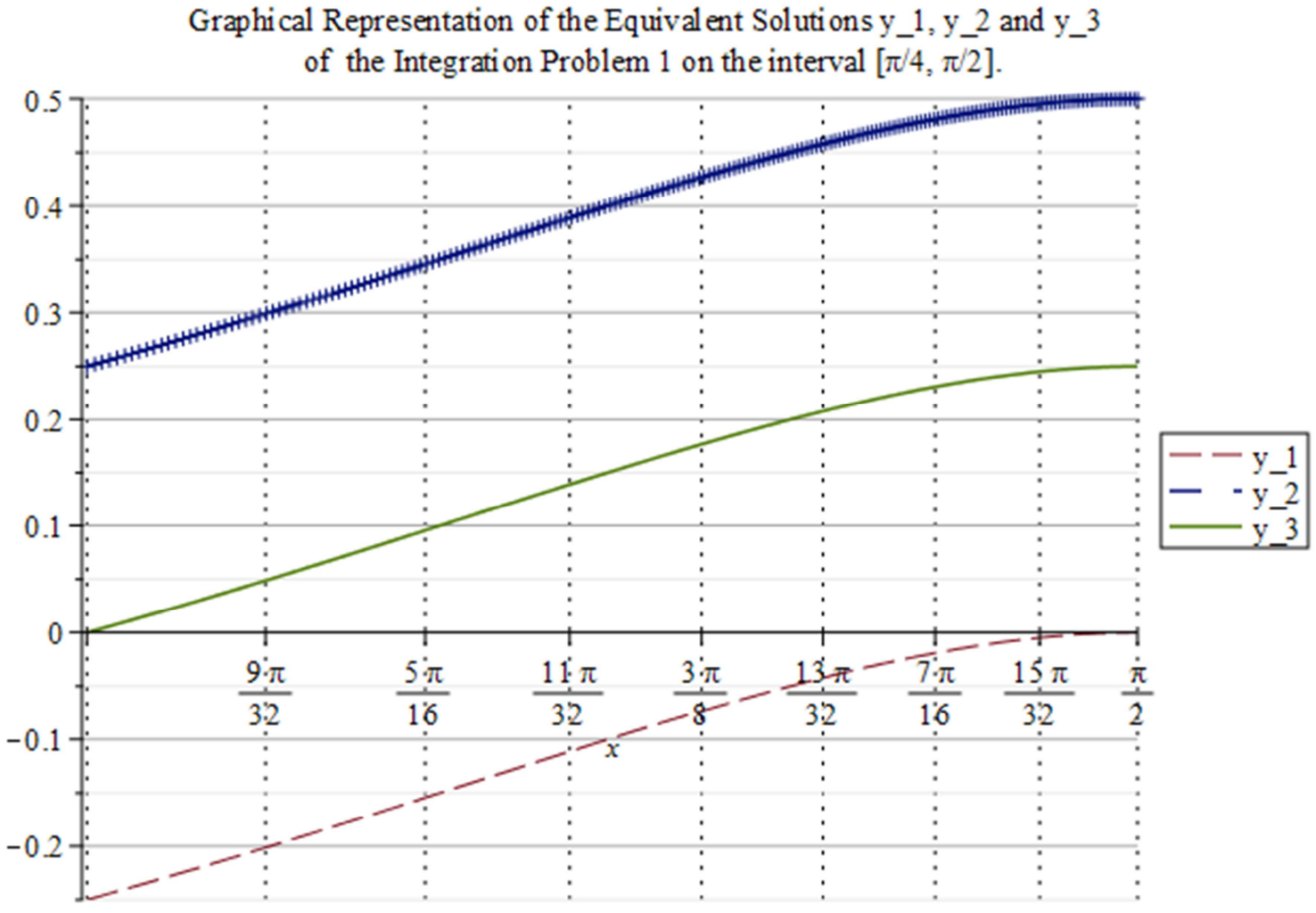

Figure 1. The graph of the solution set $\{y 1, y 2, y 3\}$ in $[0, \pi / 2]$.

$$
y_{3}=\frac{1}{4} \text {. }
$$

From the numerical result of the three solution set $\left\{y_{1}, y_{2}, y_{3}\right\}$ of the integration problem 1 given as $\int \sin x \cos x d x$, we can see that $y_{1}=y_{2}=y_{3}=1 / 4$. This without loss of generality showed clearly the existence of an equivalent solutions to an integral problem.

\subsection{Graphical Representation of the Solution Set}

Theorem 4: Let $f(x)$ be an integrable function over an interval $[\mathrm{a}, \mathrm{b}]$, there exists at least solutions set $\{y 1, y 2\}$ which are equal in numerical value evaluated on $[a, b]$ but differ in constant of integration only.

Proof: The discussion on problem 1 above is a justification for the proof of Theorem 2 .

\section{Result Discussions}

The numerical value of the solution set $\{y 1, y 2, y 3\}$ returned the same result in the Integral Calculus problem 1 shown above in section 3 . This shows the evidence of parity (equivalence) in the solution set to the integral problem. 


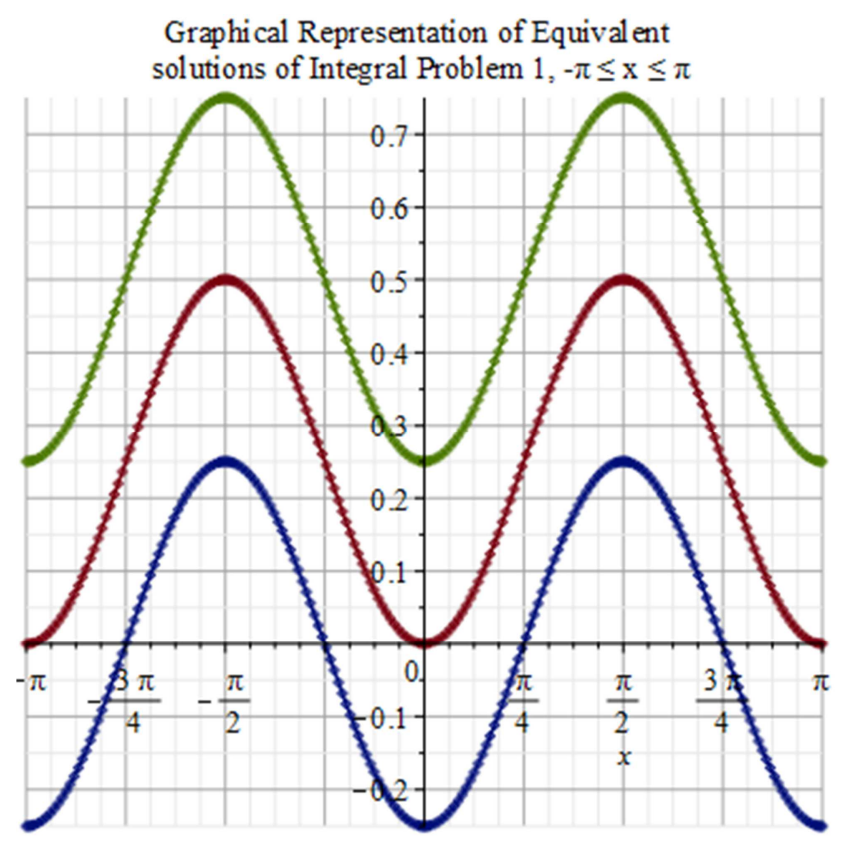

Figure 2. The solution curves $y_{1}, y_{2}$ and $y_{3}$ on $[-\pi, \pi]$.

Graphical Representation of Equival ent solutions of Integral Problem $1,-\pi / 2 \leq \mathrm{x} \leq \pi / 2$

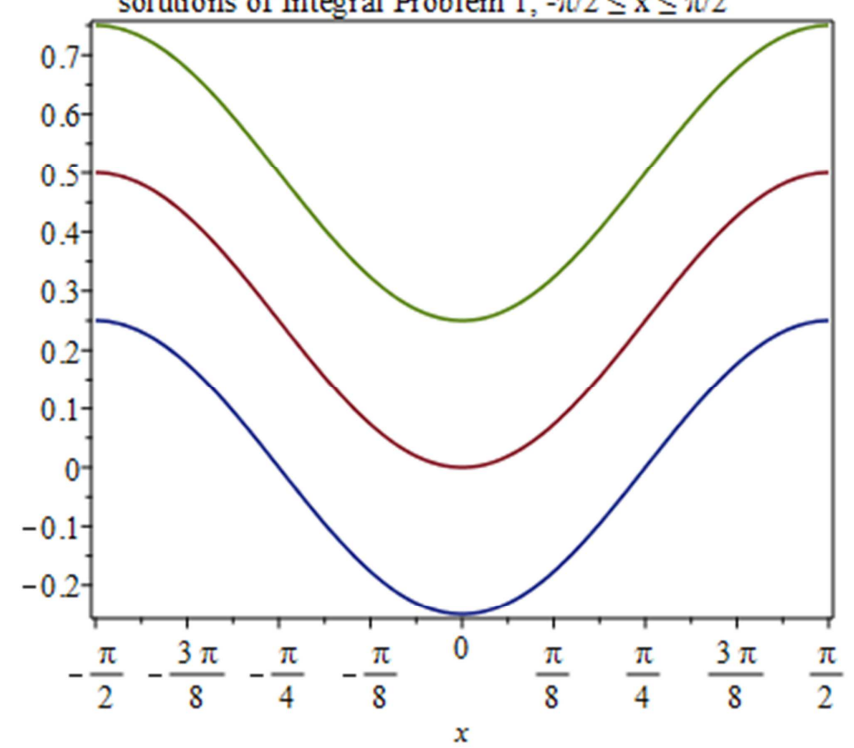

Curve $1-$ Curve $2-$ Curve 3

Figure 3. The graph of the solution set $\{y 1, y 2, y 3\}$ in $[-\pi / 2, \pi / 2]$.

The graphical representation of the solution set in the figures 1-4 on various intervals shows that the solution set $\{y 1, y 2, y 3\}$ only differs in constant of integration but are equivalent to each other. This confirms the fact that the integral of functions is not unique. For example, in figure 1, we can see that the curves are in the same direction at same distance apart but intersected the vertical axes at different value which denotes the constant of the integral of the function. The constants $c$ for $y 1, y 2, y 3$ are $0.25 .-0.25$ and 0 respectively. Likewise in the other Figures $2-4$ on their respective intervals, one can see that the curves have the same crest and troughs, the only observable difference is the $y$-intercept of the curves which denotes the difference in constant of integration.

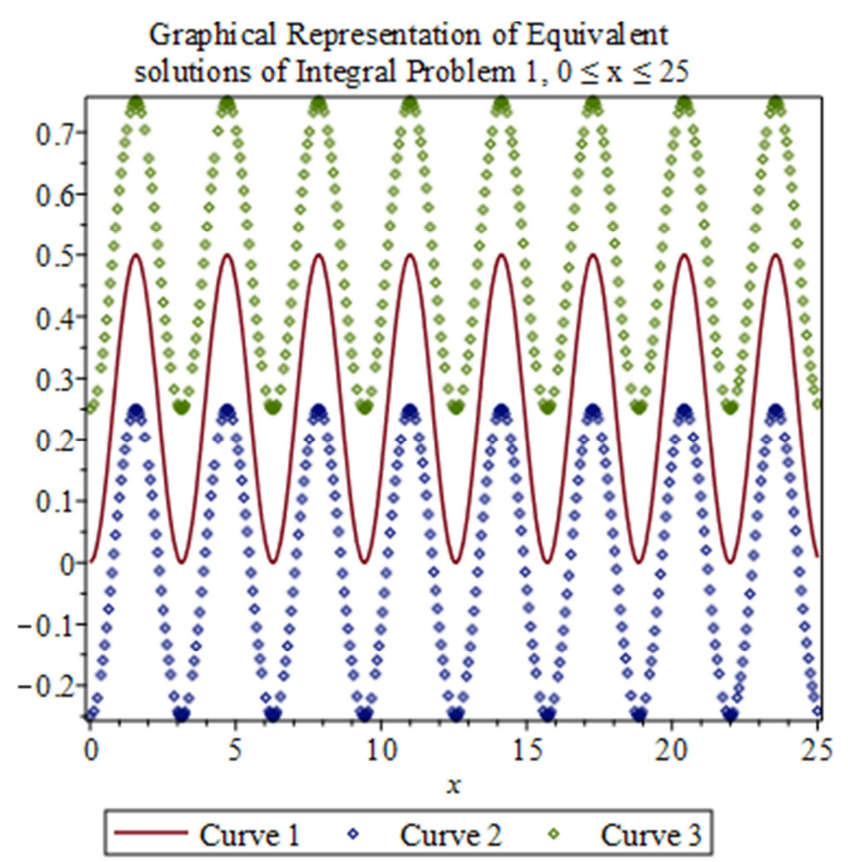

Figure 4. The plot of the solution set $\{y 1, y 2, y 3\}$ in $[0,25]$.

\section{Conclusion}

In this paper, we have been able to show the existence of an equivalent solution set to the integral of a continuous function by obtaining the numerical value of the solution set at the same specified interval. In other words, we evaluated the solution of the definite integral of the given integrand over a specified interval of integration carried out via various integration techniques and under different assumptions. The result shows the evidence of the presence of similarity in the solution set as we obtained the same numerical value. We further demonstrated the graphical representation of the solution set to see the behavior of the system of solutions obtained. The graphs displayed in the figures 1-4 were produced using Maple. The graphs further shows that solutions obtained in integral calculus are not always unique but possible to have equivalent solution set with only observable feature of difference in constant of integration.

\section{References}

[1] Jr., W. P., \& Miller, J. D. Pathways to an engineering career. Peabody Journal of Education, 2012, 87(1), 46-61.

[2] Ebele C. Okigbo and Abigail M. Osuafor, Effect of using mathematics laboratory in teaching mathematics on the achievement of mathematics students. Educational Research and Review, 2008, Vol.3(8), pp.257-261, http://www.academicjournals.org/ERR ISSN 1990-3839.

[3] Robert Wrede, and Murray R. Spiegel, Advanced Calculus, Third Edition, Schaum's Outline Series, McGraw-Hill Companies, Inc. 2010. 
[4] Tuan, S. A. and Effandi, Z. Enhancing Students' Understanding in Integral Calculus through the Integration of Maple in Learning. Procedia - Social and Behavioral Sciences 102 (2013) $204-211$.

[5] Mark Zegarelli, Calculus II for Dummies, $2^{\text {nd }}$ Edition, 2012.

[6] Leah, Edelstein-Keshet, Integral Calculus: Mathematics 103, University of British Columbia, 2010, pp. 1-234.

[7] Brian, S. Thompson. The Calculus Integral, ClassicalRealAnalysis.com (2010), [ISBN 1442180951].
[8] Mary, Barnes, Introduction to Integration Part 2: The Definite Integral, University of Sydney, 1999, pp. 1-26.

[9] Miguel A. L., Notes on Calculus II Integral Calculus, Northwestern University, Fall 2002. http://www.math.northwestern.edu/ mlerma/courses/math21 4-2-02f/.

[10] www.kshitj-iitjee.com/maths/integrals/properties-of-definiteintegrals.aspx 